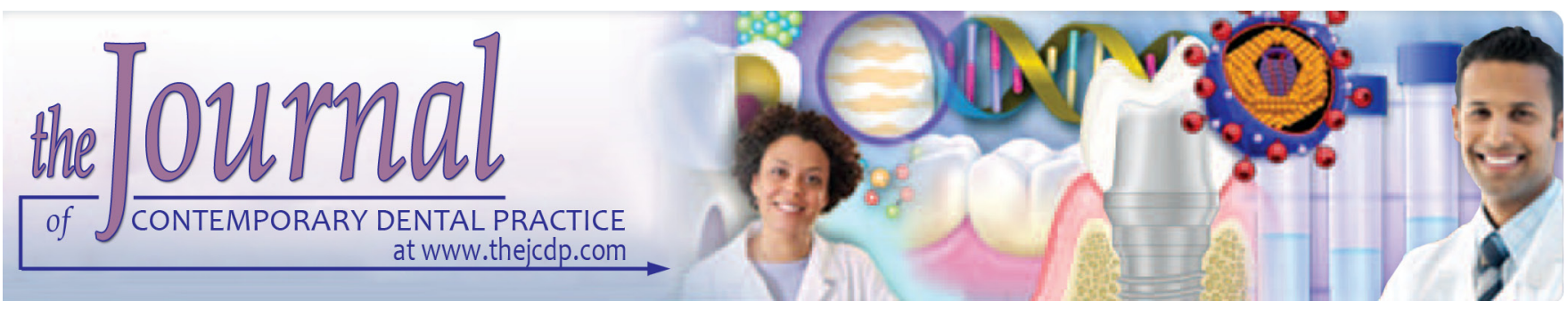

\title{
Semidirect Restorations in Multidisciplinary Treatment: Viable Option for Children and Teenagers
}

\author{
${ }^{1}$ Mateus Rodrigues Tonetto, ${ }^{2}$ Fausto Frizzera, ${ }^{3}$ Monica Barros da Silva, ${ }^{4}$ Shilpa H Bhandi, ${ }^{5}$ Milton Carlos Kuga \\ ${ }^{6}$ Kamila Figueiredo Pereira, ${ }^{7}$ Célia Regina Maio Pinzan-Vercelino, ${ }^{8}$ Matheus Coelho Bandéca
}

\begin{abstract}
Due to the esthetic necessity required nowadays, the multidisciplinar treatment became a fundamental step in the restoration success. When the patient exhibits dental agenesis of one or more elements, he can show difficulty in social interactions. The age of the patient is a limiting factor to esthetic procedures, however, it should be evaluated as a real indicative with each case. The utilization of semi-direct restorations is a viable option due the cost, esthetic and improvement of physical and mechanical properties. The purpose of this paper is to present a case detailing the confection and cementation of anterior semi-direct restorations aimed at an anatomic reestablishment associated with integrated treatment with periodontics and orthodontics.
\end{abstract}

Keywords: Indirect-direct restoration, Multidisciplinary approach, Dental agenesis, Esthetic dentistry, Dental esthetics, Permanent dental restoration.

How to cite this article: Tonetto MR, Frizzera F, da Silva MB, Bhandi SH, Kuga MC, Pereira KF, Pinzan-Vercelino CRM, Bandéca MC. Semidirect Restorations in Multidisciplinary Treatment: Viable Option for Children and Teenagers. J Contemp Dent Pract 2015;16(4):280-283.

Source of support: Nil

Conflict of interest: None

${ }^{1}$ Department of Postgraduate Program in Dentistry, University of Cuiabá, Cuiaba, Mato Grosso, Brazil

${ }^{2,5,6}$ Department of Restorative Dentistry, Araraquara Dental School, Univ Estadual Paulista, Araraquara, Sao Paulo, Brazil

${ }^{3,7,8}$ Department of Postgraduate Program in Dentistry, CEUMA University, Sao Luis, Maranhao, Brazil

${ }^{4}$ Department of Conservative Dentistry and Endodontics, MS Ramaiah Dental College and Hospital, Bengaluru, Karnataka India

Corresponding Author: Matheus Coelho Bandeca, Head Rua Jossue Montello, 01, Renascenca, Sao Luis, Maranhao, Brazil e-mail: mbandeca@gmail.com

\section{INTRODUCTION}

A harmonious smile with satisfactory cosmetic and functional outcome to the patient often depends on the multidisciplinary association of treatments, ranging from orthodontics, restorative dentistry, oral surgery and periodontics. ${ }^{1}$ Success depends on the correct rehabilitation planning. ${ }^{2,3}$ The shape, color, alignment and symmetry of the smile, teeth and gums should be carefully considered through a reverse treatment plan. ${ }^{4-6}$

For agenesis diagnostic of the maxillary lateral incisors, lack or non-formation of some elements are more often associated with genetic, ${ }^{7}$ and the 3rd molars are most affected, followed by the 2nd premolars and maxillary lateral incisors, bilaterally or not. ${ }^{4}$ The treatment has favorable prognosis if the problem is identified early. Two main treatment options can be considered depending on the treatment plan, it may be necessary to create a space to install an implant or eliminate/diminish the edentulous space and perform restorative procedures. ${ }^{4,7}$

Complex rehabilitations as faceting or full cera-mic restorations for anterior reanatomization are contraindicated in children and adolescents due to oral and facial development, complexity, longevity and cost of treatment. ${ }^{7-9}$ Thus, the use of semi-direct restorations present themselves as a viable alternative, it has a lower cost, presents the advantage of extraoral polymerization controlling, possibility of extraoral esthetic refinement and facilitated repair or adjustments, giving an option for esthetically rehabilitate a child or adolescent, which in the near future will need a new treatment due to facial alterations. $^{9-11}$

Therefore, this study aimed to describe the use of semi-direct restorations in the esthetic restoration of adolescents with agenesis of lateral incisors based on multidisciplinary treatment. 


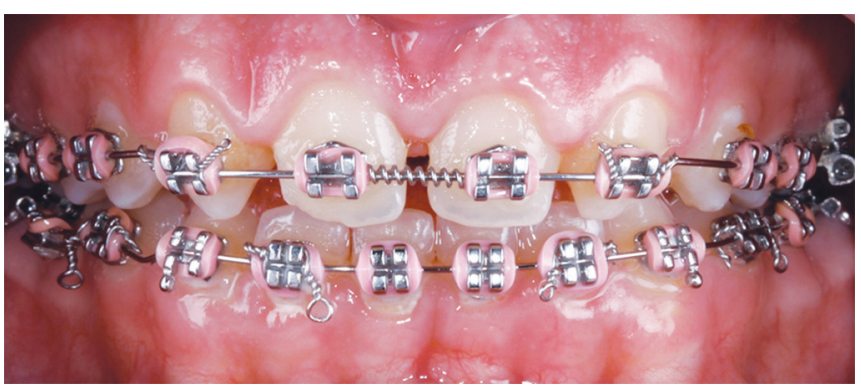

Fig. 1: Frontal view of initial case

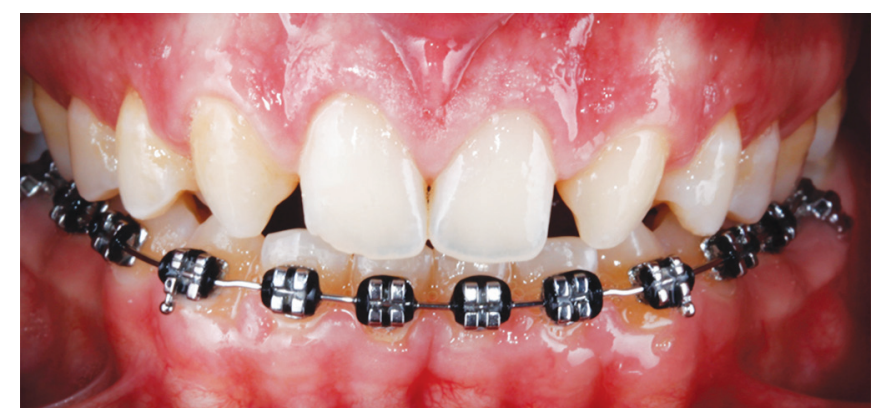

Fig. 3: Frontal view after increase clinical crown

\section{DESCRIPTION OF CASE}

A 14-year-old male patient complained of the appearance of his smile (Fig. 1). After careful evaluation, a reverse planning was performed with digital resources with intra and extra oral photographs, where it was measured through drawing of lines in his smile on his face and what would be the final result. After photographic protocol, digital planning and study model, it was decided by the multidisciplinary association in esthetic rehabilitation of the treatment of laterals incisors agenesis. In orthodontic treatment, the spaces have been reduced so that the surgery for increase of clinical crown becomes less invasive because there is the possibility of symmetry dental without root exposure during surgery. With increased of clinical crown, the restorative stage becomes simpler in execution, giving closure spaces more harmoniously through the semi-direct restorations.

The orthodontic finishing was performed with tractioning and mesialization incisors to similarity of the midline face with his teeth (Fig. 2). In order to reduce gingival exposure and increase clinical crown length, a periodontal procedure was carried out. A flapless crown lengthening procedure was performed on anterior maxillary teeth and a conventional intrasulcular flap was raised in the posterior region to remove excessive volume of the buccal bone. The gingival margin was surgically repositioned according to restorative treatment plan, at both maxillary central incisors the gingival tissue was removed to create a proportion of $10 \mathrm{~mm}$ in height and $8 \mathrm{~mm}$ in length. The clinical crowns of the remaining maxillary teeth (2nd premolars to 2nd premolars) were

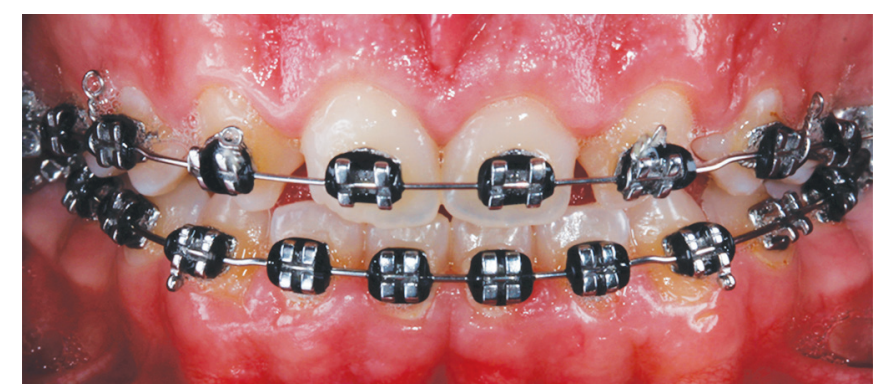

Fig. 2: Frontal view after orthodontic movement

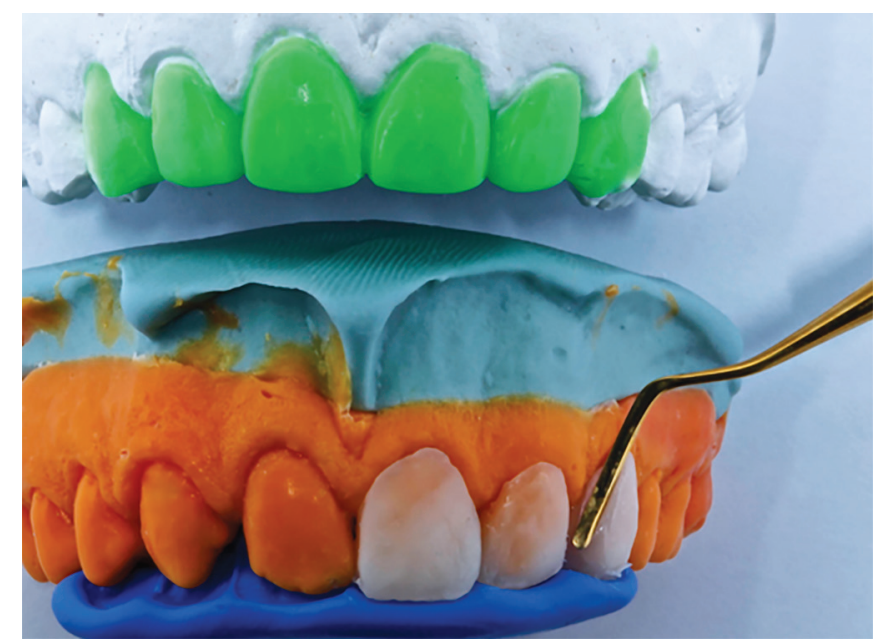

Fig. 4: Preparation of semidirect restorations according to the diagnostic waxing

operated using the maxillary central incisors as references. A distance of $2 \mathrm{~mm}$ was established between the desired gingival margin and the bone crest. The bone from the anterior region was removed through the sulcus with a delicate bone chisel. As for the posterior region rotary instruments (round bur-3018HL, KG, etc.) were used to reduce bone thickness and final adjustments were performed with hand instruments.

The cast was created after 90 days of surgical healing (Fig. 3) using alginate (Hydrogum 5, Zhermack) and the mold was filled with light and heavy silicon (Oranwash $\mathrm{L}$, indurent Gel and Zetaplus, Zhermack). The waxing diagnostic was obtained with a silicone guide to determine the size, palatal and incisal form. With the overlap the guide on the model of silicon, it was possible to make the restorations with composite enamel A1 (Premisa-Kerr Corporation, Orange, CA, USA), accompanied the proportions desired size and shape (Fig 4). The polymerization was carried out with the LED unit (DEMI Ultra-Kavo Kerr Group, USA) for 40 seconds at each increment. After completion of the restoration, the finishing of the pieces was performed with disks (Super Tray-Kerr Corporation, Orange, CA, USA) in different granulations to fit contours, anatomy and macrotexture.

The cleaning of dental elements was performed with rubber cup, and thereafter the adhesive system 


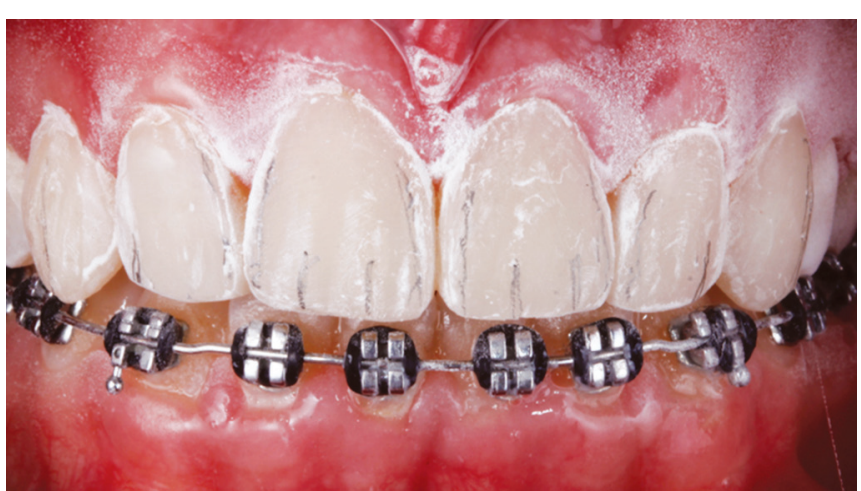

Fig. 5: Demarcation with graphite pencil of the areas that should receive more refined finish

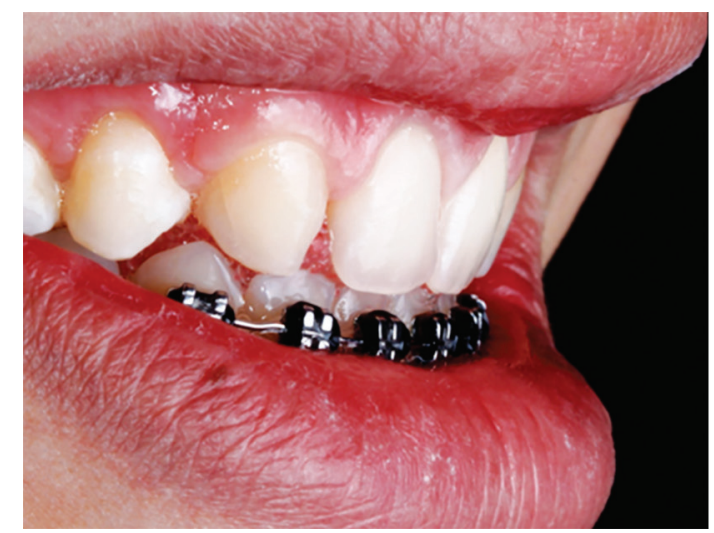

Fig. 7: One-year follow-up of semiprofile compared to the initial situation

(OptiBond S-Kerr Corporation, Orange, CA, USA) was applied. Transparent matrix and teflon tape were used to protect adjacent teeth and then $37 \%$ phosphoric acid was carried out for 30 seconds throughout the enamel. The adhesive was applied to the full extension of the teeth, according to the manufacturer and light cured for 20 seconds each tooth.

The restorative pieces were conditioned with the same acid for 30 seconds and a thin layer of adhesive (OptiBond S-Kerr Corporation, Orange, CA, USA) was applied. Cementation was performed with flowable composite (Premisa flowable-Kerr Corporation, Orange, CA, USA) due to the small thickness of restorative pieces presented, enabling their proper curing and filling of possible defects. The excesses were removed with the aid of a dental explorer and each face was light cured for 40 seconds. The finishing was performed again using disks (Super Tray-Kerr Corporation, Orange, CA, USA) (Fig. 5), occlusal and interproximal contacts checked, final polishing was completed (Fig. 6), providing functionality and esthetics at 1 year follow-up (Fig. 7).

\section{DISCUSSION}

The treatment approach in dentistry has changed over the years and nowadays dentistry is not only curative, corrective and functional but also esthetic. ${ }^{8,9}$ It is necessary the

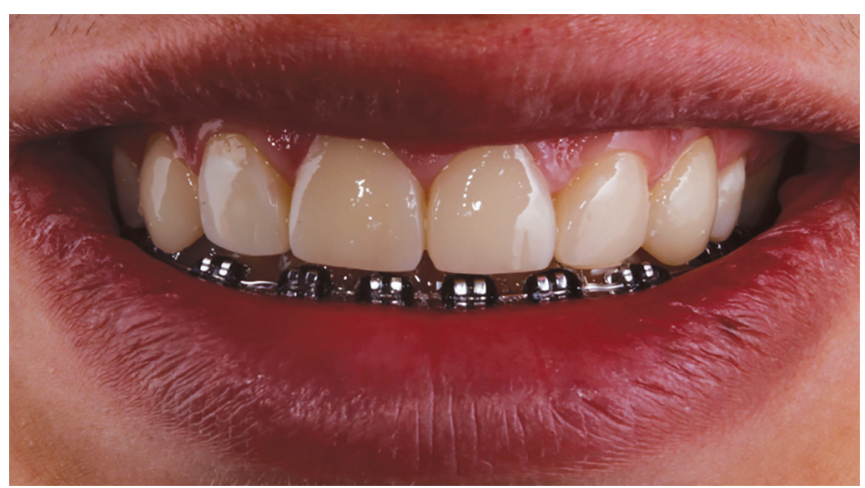

Fig. 6: Final front view smiling

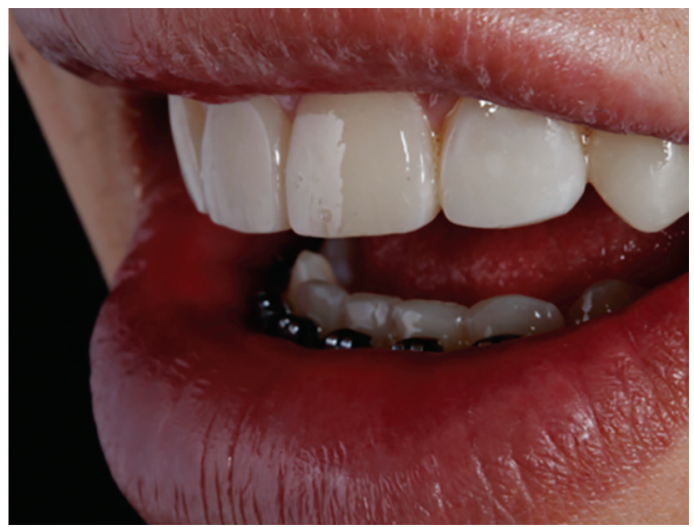

integration between areas to respond effectively to the individual needs of each patient. ${ }^{4}$ The inter-relationship between orthodontics, periodontics and dentistry were essential to resolve the reported case. With orthodontic completion incisors were mesializated and thus reducing the interdental spaces, allowing a less invasive surgical to increase of crown, since teeth do not need large vertical increases because the horizontal spaces have been reduced, ensuring adequate proportion without any increase in crown root level.

Lateral incisors agenesis is among the most common anomalies involving dental elements ${ }^{7}$ and it can affect the mental well being of children and teenagers unhappy with their smile. Among the treatment options, it can be considered installation of implants and/or orthodontic movements and subsequent oral rehabilitation. ${ }^{4,7}$ Teenagers are undergoing oral and facial development and ceramics restorations for esthetical purposes are usually not indicated since it would present a reduced lifespan requiring their replacement after facial growth. ${ }^{7,8}$ Rehabilitation with composites requires more technical work sensitivity, ${ }^{9}$ allowing the use of semi-direct technique.

Semi-direct adhesive restorations constitute an excellent esthetic alternative for both anterior as posterior teeth. The possibility of eliminating the drawbacks related to the use of composite resin for direct technique is the biggest indicator of success in this restorative 
procedure. ${ }^{13,16,17}$ The option to work with semi-direct restorations has several advantages mainly to rehabilitate children or teenagers. The intraoral time for treatment is reduced, avoiding possible technical gaps providing greater contribution from the patient. ${ }^{12}$ The polymerization is improved and the polymerization shrinkage of the restoration occurs outside the mouth, reducing marginal gaps., ${ }^{9,13}$ Another advantage is the way of insertion of composite resin outside the mouth, with ease of viewing and handling to draw the anatomy and contact point for each element. ${ }^{14,15}$ As we were not performed, the thickness of the restoration was reduced, thus opting to use only one color of enamel resin.

Because of few studies of this promising technique in the literature, it becomes evident the need for investigations and longitudinal follow-up of the cases already implemented. Its indication has to be supported scientifically in addition to increase understanding of the interaction with the remaining tooth.

\section{ACKNOWLEDGMENTS}

The integration between areas is necessary to respond effectively to the individual needs of each patient, and the utilization of semi-direct restorations is a viable option due the cost, esthetic and improvement of physical and mechanical properties. Furthermore, this technic has several advantages mainly to rehabilitate children or teenagers, since the intraoral time for treatment is reduced, avoiding possible technical gaps providing greater contribution from the patient.

\section{REFERENCES}

1. Polat ZS, Tacir IH. Esthetic rehabilitation of avulsed-replanted anterior teeth: a case report. Dent Traumatol 2008;24: 385-389.

2. Spear FM, Kokich VG. A multidisciplinary approach to esthetic dentistry. Dent Clin North Am 2007;51:487-505.
3. Aron VO. Porcelain veneers for primary incisors: a case report. Quintessence Int 1995;26:455-457.

4. Krassnig M, Fickl S. Congenitally missing lateral incisors: a comparison between restorative, implant, and orthodontic approaches. Dent Clin North Am 2011;55:283-299.

5. Goodlin R. Photographic-assisted diagnosis and treatment planning. Dent Clin North Am 2011;55:211-227.

6. Calixto LR, Yoshio I, Eustáquio J, Bandeca MC, Andrade MF. Photography of dental protocol for communication between dentist and dental technicians in indirect restorations. Dental Press Estet 2011:42-50.

7. Fugazzotto PA. Evidence-based decision making: replacement of the single missing tooth. Dent Clin North Am 2009; 53:97-129.

8. Chu FC, Yim TM, Wei SH. Clinical considerations for reattachment of tooth fragments. Quintessence Int 2000;31: 385-391.

9. Tonolli G, Hirata R. Semi-direct technique for the restoration of posterior teeth: a treatment alternative. Rev Assoc Paul Cir Dent 2010;1:90-96.

10. Bagis $\mathrm{YH}$, Rueggeberg FA. The effect of post-cure heating on residual, unreacted monomer in a commercial resin composite. Dent Mater 2000;16:244-247.

11. Bagis $\mathrm{YH}$, Rueggeberg FA. Effect of post-cure temperature and heat duration on monomer conversion of photo-activated dental resin composite. Dent Mater 1997;13:228-232.

12. Tay FR, Wei SH. Indirect posterior restorations using a new chairside microhybrid resin composite system. J Adhes Dent 2001;3:89-99.

13. Roeters JJ. Extended indications for directly bonded composite restorations: a clinician's view. J Adhes Dent 2001;3:81-87.

14. Bandeca MC, Tonetto MR, Barros ED, Souza Pinto SC, Firoozmand LM, Andrade MF, Saad JRC, Maia Filho EM, De Sousa Queiroz RC. Indirect resin onlay cemented with selfadhesive resin cement: a comprehensive clinical overview. W J Dent 2012;3:273-277.

15. Ewoldsen N, Sundar V, Bennett W, Kanya K, Magyar K. Clinical evaluation of a visible light-cured indirect composite for long-term provisionalization. J Clin Dent. 2008;19(1):37-41.

16. Kim H, Song MJ, Shin SJ, Lee Y, Park JW. Esthetic rehabilitation of single anterior edentulous space using fiber-reinforced composite. Restor Dent Endod 2014;39(3):220-225.

17. Magne P. Composite resins and bonded porcelain: the postamalgam era. J Calif Dent Assoc 2006;34(2):135-147. 